\title{
Collagen synthesis and gap junctions: the highway for metastasis of ovarian cancer
}

\author{
Yinfeng Lyu ${ }^{1} \cdot$ Chenchen Feng $\mathbb{D}^{1}$
}

Received: 3 January 2021 / Revised: 14 January 2021 / Accepted: 15 January 2021 / Published online: 18 February 2021

(c) The Author(s), under exclusive licence to United States and Canadian Academy of Pathology 2021

Prognosis of ovarian cancer depends on local invasion and metastasis. The extracellular matrix (ECM) plays a critical role in the tumor microenvironment. The recent study by Guo et al. in Laboratory Investigation comprehensively explores the role of the collagen-stabilizing PLOD enzymes in ovarian cancer. The authors show that PLOD3 appears to play major role in the prognosis and metastasis of ovarian cancer and is strongly correlated with the expression of gap junction alpha-1 protein (GJAl; also known as connexin-43 (CX43)). If extracellular collagen serves as the highway for tumor metastasis, gap junctions are the signposts and the gas stations. The findings by Guo et al. strongly support the role of $P L O D$ in the invasion and metastasis of ovarian cancer. One critical question is how cells initiating metastasis can sense the readiness of the ECM, perhaps this is done via communication by gap junctions. Such regulatory signaling warrants investigation and Guo's work reveals the first piece of the puzzle by revealing the possible interaction between PLOD3 and GJA1.

Prognosis of ovarian cancer depends on by local invasion and metastasis, which incorporates the interplay of multiple factors, including alterations in gene copy number and expression levels that result in alterations of multiple signaling pathways and disruption of tumor microenvironment.

The ECM plays a critical role in the tumor microenvironment. During the invasion of ovarian cancer, the ECM undergoes significant structural remodeling, mainly characterized by collagen deposition, increases in fibronectin and proteoglycans, and enhanced cross-linking of the matrix [1]. The ECM can facilitate tumor progression through interaction between its structure and signaling pathways. Certain changes in the synthesis, maturation,

Chenchen Feng

fengchenchen@huashan.org.cn

1 Department of Urology, Huashan Hospital, Fudan University, Shanghai, China orientation, and cross-linking of collagen in ECM promote tumor invasion and metastasis [2]. Cancer cells reshape extracellular collagen as they lay highways and use them to spread to distant sites. Nonetheless, specific mechanism of how extracellular collagen promotes tumor metastasis remains elusive. It is thus important to gain insight into the relationship between collagen alterations in the tumor microenvironment and tumor metastasis.

The study by Guo et al. [3], published in this issue, comprehensively explores the role of the PLOD family in ovarian cancer. Collagen is a major component of the ECM, and its synthesis is modulated by the collagen-stabilizing $P L O D$ family of enzymes. The procollagen $\alpha$ chain is the basic unit of collagen and its most common motif is Gly-X$\mathrm{Y}$ (X, proline and $\mathrm{Y}$, hydroxyproline). During collagen synthesis, Y must undergo lysyl hydroxylation by $P L O D$ encoded lysyl hydroxylase (LH) to generate triple-helix procollagen from the $\mathrm{C}$-terminal to the $\mathrm{N}$-terminal before being secreted into the ECM for further non-helical end excision, glycosylation, and/or cross-linking to form stable collagenous bundles [4]. Guo et al. report the overexpression of $P L O D$ genes in ovarian cancer and its impact on clinical outcome. The expression of PLOD2 and PLOD3 is higher in metastatic lesions, and overexpression of $P L O D 3$ is closely related to the staging of advanced tumor and reduced overall survival.

In ovarian cancer, invasion and metastasis determine prognosis. High expression of PLOD enzymes can hydroxylate the terminal peptide lysine, promote the cross-link of collagen and increase the consolidation of the matrix, so as to pave the metastatic roadbed [5]. The pro-tumorigenic effect of $P L O D$ has been reported in a variety of cancers. It has been shown in gastric cancer that the PLOD3 copy number alteration is closely related to its expression levels [6]. PLOD also plays a role in tumor invasion and metastasis by promoting epithelial-mesenchymal transition (EMT). Under hypoxic conditions, PLOD2 expression is up-regulated in glioma cells, with the downregulation of E-cadherin, a marker associated with EMT. Silencing 
PLOD2 can reverse expression of hypoxic genes, suggesting that PLOD2 promotes hypoxia-induced EMT [7].

Guo et al. propose that PLOD3 plays major role in prognosis and metastasis in ovarian cancer. The LH3 protein encoded by PLOD3 has a specific activity of galactosyl hydroxylysine glucosyltransferase and hydroxylysyl galactosyltransferase (GLT) in addition to the modification to lysine hydroxylation, which promotes the high-level glycosylation of type IV and type VI collagen [8]. Posttranslational glycosylation of collagen is a crucial step in collagen biosynthesis, but the mechanism remains to be explored. Glycosyl transfer 25 domain 1 (GLT25D1 or COLGALT1) and lysine hydroxylase 3 (LH3, PLOD3) have been reported to co-localize in hepatocellular carcinoma cells and modify the collagen substrate mannose-binding lectin [9]. Also, LH3 serves as a docking molecule in tumor microenvironment via binding to pro-matrix metalloproteinase (MMP)-9 fibronectin-like domain, which is recruited from the matrix, activated and translocated onto the surface of fibroblasts. Activated MMP-9 can further induce TGF- $\beta$, which promotes fibroblast differentiation into myofibroblasts, reshapes ECM, and accelerates tumor progression [10]. Guo's study also reveals that $P L O D 3-\mathrm{KD}$ significantly inhibits the proliferation of ovarian cancer. Likewise, studies in glioma have also shown that PLOD3 silencing inhibits proliferation and induces $\mathrm{G} 1$ phase arrest by regulating the p53-independent p21 pathway [11]. These results suggest that PLOD3 may also play a role in cell cycle regulation.

Guo et al. point out that the expression of $P L O D 3$ in ovarian cancer is strongly correlated with that of GJAl (CX43), which is intriguing. Connexins (CXS) are the molecular basis of the gap junction, among which the CX43 is the most widely distributed and most closely related to cancer. CX43 consists of six dumbbell-shaped cross-membrane CXS that forms a hydrophilic pore. A gap junction is formed between both sides of the cells by connecting CXS end-to-end on the membrane, allowing small molecules like water, ions, metabolites, second messengers, antigens, and even microRNAs to pass through $[12,13]$, which is referred to as gap junction intercellular communication. CX43 has previously been recognized as a potential tumor suppressor as most studies show low CX43 expression in cancer. Nevertheless, recent papers demonstrate that CX43 plays important roles in facilitating metastasis. Tang et al. [14] reported low expression of CX43 in primary gastric cancer tissues, but the expression level of CX43 increased significantly in invading cancer cells in the peritoneum and peritoneal metastatic tumor tissue, suggesting that exogenous overexpression of CX43 plays an important role in promoting metastasis from gastric area to the peritoneum. The expression of CX43 in glioma cells is significantly increased by low dose $\gamma$-rays, and the activation of p38 promotes the migration of glioma cells. Downregulating

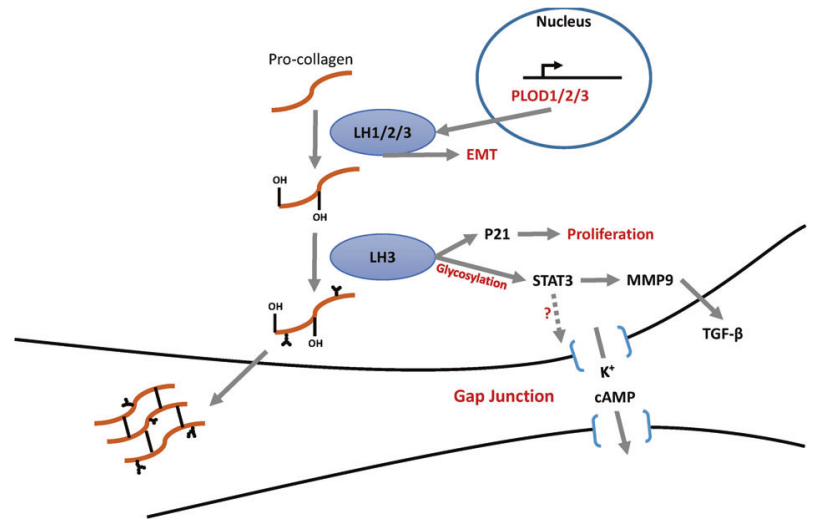

Fig. 1 PLODs links collagen metabolism and gap juntion in ovarian cancer. Schematic showing established and postulated regulatory signaling between $P L O D$, collagen synthesis, connexin, and gap junctions.

CX43 inhibits both migration and the activation of p38 [15]. Moreover, invasiveness of prostate cancer cells is increased with overexpression of CX43 and SNAI1, and vice versa. This implies that CX43 interacts with SNAI1 with a feedback loop that promotes the invasion and metastasis [16]. In healthy ovarian tissue, CX43 is regulated by gonadotropin at the ranscriptional, translational, and posttranslational levels, regulating follicular and oocyte formation. In ovarian cancer, CX43 is reported to be downregulated in some studies, and upregulation of gap junction could inhibit cancer growth [17]. In studies of metastatic ovarian cancer, however, the dual nature of CX43 has also been demonstrated. Those studies suggest that have CX43 plays a key role in ovarian cancer migration, possibly as a downstream effector by TGF- $\beta$ through Smad2/3 signaling [18].

Thus, if extracellular collagen serves as the highway for tumor metastasis, gap junctions are the signposts and the gas stations. CX43 not only transmits important information, but also acts as a material supply channel in the process of tumor metastasis. Guo et al. have for the first time proposed a possible association between the PLOD enzymes and CX43. This finding is of importance in understanding the initiation and progression of tumor metastasis. It is therefore logical to surmise that when extracellular microenvironment, such as the maturation and cross-linking of extracellular collagen are suitable, expression of gap junction proteins will increase for communication and the energy supply necessary for metastasis to occur. In line with previous studies that have shown interaction between collagen synthesis and gap junctions [19], more insightful mechanistic analysis is warranted. Of note, PLOD3 and STAT3 pathways are significantly associated with metastasis in lung cancer. Functional analysis shows that PLOD3 interacts with STAT3 to induce expression of MMP-2 and MMP-9 to enhance tumor metastasis [20]. Intracellular protein levels of CX43 can be increased via 
STAT3 signaling following tyrosine kinase 2 activation of angiotensinogen II in rat kidney [21], suggesting a possible regulatory role of STAT3.

In conclusion, the findings by Guo et al. strongly support the role of $P L O D$ in invasion and metastasis of ovarian cancer. One critical question is how cells initiating metastasis can sense the readiness of ECM, possiblly via communication by gap junction. Such regulatory signaling warrants investigation and Guo's work reveal the first piece in the puzzle by revealing the possible interaction between PLOD3 and CX43 (Fig. 1).

Funding This study was sponsored in part by National Natural Science Foundation of China (Grant no. 81874123).

\section{Compliance with ethical standards}

Conflict of interest The authors declare no competing interests.

Publisher's note Springer Nature remains neutral with regard to jurisdictional claims in published maps and institutional affiliations.

\section{References}

1. Natarajan S, Foreman KM, Soriano MI, Rossen NS, Shehade H, Fregoso DR, et al. Collagen remodeling in the hypoxic tumormesothelial niche promotes ovarian cancer metastasis. Cancer Res. 2019;79:2271-84.

2. Han W, Chen S, Yuan W, Fan Q, Tian J, Wang X, et al. Oriented collagen fibers direct tumor cell intravasation. Proc Natl Acad Sci USA. 2016;113:11208-13.

3. Guo T, Gu C, Li B et al. PLODs are overexpressed in ovarian cancer and are associated with gap junctions via connexin 43. Lab Investig. 2021. https://doi.org/10.1038/s41374-021-00533-5.

4. Shao S, Zhang X, Duan L, Fang H, Rao S, Liu W, et al. Lysyl hydroxylase inhibition by minoxidil blocks collagen deposition and prevents pulmonary fibrosis via TGF-beta(1)/Smad3 signaling pathway. Med Sci Monit. 2018;24:8592-601.

5. van der Slot AJ, Zuurmond A-M, van den Bogaerdt AJ, Ulrich MMW, Middelkoop E, Boers W, et al. Increased formation of pyridinoline cross-links due to higher telopeptide lysyl hydroxylase levels is a general fibrotic phenomenon. Matrix Biology. 2004;23:251-7.

6. Cheng L, Wang P, Yang S, Yang Y, Zhang Q, Zhang W, et al. Identification of genes with a correlation between copy number and expression in gastric cancer. BMC Med Genom. 2012;5:14.
7. Song Y, Zheng S, Wang J, Long H, Fang L, Wang G, et al. Hypoxia-induced PLOD2 promotes proliferation, migration and invasion via PI3K/Akt signaling in glioma. Oncotarget. 2017;8:41947-62.

8. Qi Y, Xu R. Roles of PLODs in collagen synthesis and cancer progression. Front Cell Dev Biol. 2018;6:66.

9. Liefhebber JM, Punt S, Spaan WJ, van Leeuwen HC. The human collagen beta(1-O)galactosyltransferase, GLT25D1, is a soluble endoplasmic reticulum localized protein. BMC Cell Biol. 2010;11:33.

10. Dayer C, Stamenkovic I. Recruitment of matrix metalloproteinase-9 (MMP-9) to the fibroblast cell surface by lysyl hydroxylase 3 (LH3) triggers transforming growth factor-beta (TGF-beta) activation and fibroblast differentiation. J Biol Chem. 2015;290:13763-78.

11. Tsai CK, Huang LC, Tsai WC, Huang SM, Lee JT, Hueng DY. Overexpression of PLOD3 promotes tumor progression and poor prognosis in gliomas. Oncotarget. 2018;9:15705-20.

12. Esseltine JL, Laird DW. Next-generation connexin and pannexin cell biology. Trends Cell Biol. 2016;26:944-55.

13. Gupta A, Anderson H, Buo AM, Moorer MC, Ren M, Stains JP. Communication of cAMP by connexin43 gap junctions regulates osteoblast signaling and gene expression. Cell Signal. 2016;28: 1048-57.

14. Tang B, Peng ZH, Yu PW, Yu G, Qian F. Expression and significance of $\mathrm{Cx} 43$ and E-cadherin in gastric cancer and metastatic lymph nodes. Med Oncol. 2011;28:502-8.

15. Ghosh S, Kumar A, Tripathi RP, Chandna S. Connexin-43 regulates p38-mediated cell migration and invasion induced selectively in tumour cells by low doses of gamma-radiation in an ERK-1/2-independent manner. Carcinogenesis. 2014;35:383-95.

16. Ryszawy D, Sarna M, Rak M, Szpak K, Kedracka-Krok S, Michalik M, et al. Functional links between Snail-1 and $\mathrm{Cx} 43$ account for the recruitment of $\mathrm{Cx} 43$-positive cells into the invasive front of prostate cancer. Carcinogenesis. 2014;35:1920-30.

17. Dai S, Liu J, Sun X, Wang N. Ganoderma lucidum inhibits proliferation of human ovarian cancer cells by suppressing VEGF expression and up-regulating the expression of connexin 43. BMC Complement Altern Med. 2014;14:434.

18. Qiu X, Cheng JC, Zhao J, Chang HM, Leung PC. Transforming growth factor-beta stimulates human ovarian cancer cell migration by up-regulating connexin 43 expression via Smad2/3 signaling. Cell Signal. 2015;27:1956-62.

19. Damaraju S, Matyas JR, Rancourt DE, Duncan NA. The role of gap junctions and mechanical loading on mineral formation in a collagen-I scaffold seeded with osteoprogenitor cells. Tissue Eng Part A. 2015;21:1720-32.

20. Baek JH, Yun HS, Kwon GT, Kim JY, Lee CW, Song JY, et al. PLOD3 promotes lung metastasis via regulation of STAT3. Cell Death Dis. 2018;9:1138.

21. Li H, Spagnol G, Zheng L, Stauch KL, Sorgen PL. Regulation of connexin43 function and expression by tyrosine kinase 2. J Biol Chem. 2016;291:15867-80. 